\title{
Does oestradiol attenuate the damaging effects of a fructose-rich diet on cardiac Akt/endothelial nitric oxide synthase signalling?
}

\author{
Snjezana Romic ${ }^{1}$, Snezana Tepavcevic ${ }^{1}$, Zorica Zakula ${ }^{1}$, Tijana Milosavljevic ${ }^{1}$, Mojca Stojiljkovic ${ }^{1}$, \\ Maja Zivkovic ${ }^{2}$, Milan Popovic ${ }^{2}$, Aleksandra Stankovic ${ }^{2}$ and Goran Koricanac ${ }^{1 *}$ \\ ${ }^{1}$ Laboratory for Molecular Biology and Endocrinology, Vinca Institute of Nuclear Sciences, University of Belgrade, \\ PO Box 522, 11001 Belgrade, Serbia \\ ${ }^{2}$ Laboratory for Radiobiology and Molecular Genetics, Vinca Institute of Nuclear Sciences, University of Belgrade, \\ Belgrade, Serbia
}

(Submitted 11 January 2012 - Final revision received 17 May 2012 - Accepted 9 August 2012 - First published online 16 October 2012)

\begin{abstract}
Fructose-rich diets (FRD) cause cardiac insulin resistance manifested by impairment of Akt/endothelial NO synthase (eNOS) signalling. In contrast, oestradiol (E2) activates this signalling pathway in the heart. To study the ability of E2 to revert the detrimental effect of fructose on cardiac Akt/eNOS, female rats were subjected to a FRD and ovariectomy followed with or without E2 replacement. We also analysed the effects of the FRD and E2 on cardiac extracellular signal-regulated kinase (Erk 1/2) signalling related to their role in cardiac hypertrophy development. Expression of Akt, eNOS and Erk 1/2, as well as regulatory phosphorylations of these molecules were determined. The protein expression of cardiac Akt and eNOS was not affected by the diet or E2 treatment. However, the FRD was accompanied by a decrease in Akt phosphorylation at $\mathrm{Ser}^{473}$ and $\mathrm{Thr}^{308}$, and eNOS at Ser ${ }^{1177}$, while the phosphorylation of eNOS at Thr ${ }^{495}$ was increased. E2 replacement in ovariectomised fructose-fed rats caused a reversion of the diet effect on Akt and eNOS serine phosphorylation, but mostly had no effect on threonine phosphorylation of the molecules. The FRD and E2 treatment did not influence Erk 1/2 expression and phosphorylation and heart mass as well. The data show that E2 selectively suppress the negative effects of a FRD on Akt/eNOS signalling and probably point to the different effects of $\mathrm{E} 2$ on kinase/phosphatase pathways responsible for phosphorylation/dephosphorylation of Akt and eNOS. Furthermore, the results suggest that the heart of females in the reproductive period is partially protected against the damaging effects of increased fructose intake.
\end{abstract}

Key words: Fructose: Oestradiol: Heart: Protein kinase B: Endothelial nitric oxide synthase

Increased intake of fructose causes dramatic metabolic disturbances leading to the development of insulin resistance, type 2 diabetes and the metabolic syndrome in humans and the animals ${ }^{(1,2)}$. Although the genesis of fructose-induced disorders is very complex and includes different tissues such as hepatic, adipose, etc., cardiac events are also observed ${ }^{(3,4)}$. As has recently been reported, among other disturbances, a fructose-rich diet (FRD) is accompanied by cardiac insulin resistance that targets Akt/endothelial NO synthase (eNOS) signalling ${ }^{(5,6)}$. Consequences of enhanced fructose intake are shown to be sex-dependent and the protective effect observed in females is referred to oestrogens ${ }^{(7)}$. It is well known that oestrogens are important regulators of cardiac function and the effects of physiological concentrations of oestrogens are beneficial for the heart ${ }^{(8,9)}$.
The phosphatidylinositol 3 kinase/Akt signalling pathway regulating eNOS activity and eNOS itself is very important for cardiac physiology ${ }^{(10-12)}$. To be more interesting, Akt and eNOS are also attributed to female-specific cardiac protection $^{(13,14)}$, and they have been shown to be activated in the heart by oestrogens ${ }^{(15,16)}$ in a rapid non-genomic way ${ }^{(17)}$. Moreover, the phosphatidylinositol 3 kinase/Akt/eNOS axis is a backbone of insulin beneficial cardiac effects and a target of cardiac insulin resistance ${ }^{(18)}$. Activation of the Akt/ eNOS signalling pathway by insulin or otherwise requires the phosphorylation of Akt at serine 473 and threonine 308 positions $^{(19)}$, as well as the phosphorylation of eNOS at serine 1177 and dephosphorylation at threonine $495^{(20)}$.

In addition, there are no data that a FRD influences cardiac extracellular signal-regulated kinase (Erk 1/2) signalling $^{(6)}$. However, an increased intake of fructose is blamed to be an

Abbreviations: E2, oestradiol; eNOS, endothelial nitric oxide synthase; Erk 1/2, extracellular signal-regulated kinase; FFR, fructose-fed rats; FRD, fructoserich diet; PDK1, phosphoinositide-dependent protein kinase 1; ND, normal diet; OVX, ovariectomy; mTOR, mammalian target of rapamycin. 
inducer of heart hypertrophy development ${ }^{(21-23)}$. In contrast, oestradiol (E2) has the ability to activate cardiac mitogenic Erk $1 / 2$ signalling $^{(24,25)}$ and to prevent cardiac hypertrophy in different pathological conditions ${ }^{(26,27)}$.

On the basis of existing literature data indicating the detrimental effects of a FRD on cardiac Akt/eNOS signalling, as well as the crucial importance of the same pathway for oestrogen signalling in the heart, we hypothesised that E2 might attenuate cardiac disturbances related to an enhanced intake of fructose. We also analysed the effects of a FRD and E2 replacement on cardiac Erk 1/2 signalling related to their role in cardiac hypertrophy development. To validate our hypothesis, we analysed Akt, eNOS and Erk 1/2 expression and phosphorylation in the heart of ovariectomised female rats subjected to a FRD in the absence and presence of E2.

\section{Experimental methods \\ Chemicals}

Fructose was purchased from API-PEK (Becej). Anti-phospho$\operatorname{Ser}^{473}$ Akt, anti-phospho-Thr ${ }^{308}$ Akt, anti-phospho-Ser ${ }^{1177}$ eNOS, anti-phospho-Thr ${ }^{495}$ eNOS, anti-actin and secondary anti-mouse antibody were obtained from Santa Cruz Biotechnology, Inc. Anti-Akt antibody was purchased from Affinity Bioreagents, Inc. Anti-eNOS antibody, anti-Erk $1 / 2$ and antiphospho-Thr ${ }^{202} / \operatorname{Tyr}^{204}$ Erk $1 / 2$ were products of Cell Signaling. Reagents for the bicinchoninic acid assay were purchased from Pierce. Secondary anti-rabbit and anti-goat antibodies, insulin and 17ß-E2 were purchased from Sigma-Aldrich Corporation.

\section{Animals}

Animal experiments were conducted in accordance with standards approved by the official Vinca Institute's Ethical Committee for Experimental Animals.

Female Wistar rats (21 d old) were separated from their mothers and divided into the control (normal diet; ND) group and FRD group. The control animals had free access to tap water and standard laboratory chow. The animals on the FRD were fed by the same food, but instead of tap water they drank $10 \%(\mathrm{w} / \mathrm{v})$ fructose solution. The body weight of rats in the two experimental groups was the same at the beginning of the diet. All animals were kept under standard temperature and dark-light conditions. The diet regimen prolonged for 9 weeks. Body mass was recorded during the diet regimen, while mass of the heart was measured after killing.

At 2 weeks before killing, all animals were bilaterally ovariectomised, under ketamine $(40 \mathrm{mg} / \mathrm{kg}$, intraperitoneally)-xylazine $(5 \mathrm{mg} / \mathrm{kg}$, intraperitoneally) anaesthesia, to remove endogenous oestrogens and to prevent their effects $^{(28)}$. Then, half of the fructose-fed rats (FFR) were subjected to E2 replacement treatment $(40 \mu \mathrm{g} / \mathrm{kg}$, subcutaneously, every second day) after ovariectomy (OVX), which continued until the day before killing. To study the fructose and E2 effects on the insulin regulation of the analysed molecules, the experimental groups were divided into two subgroups, one of which was treated with insulin (12 IU/kg of body weight, $40 \mathrm{~min}$ before killing, intraperitoneally). Each group contained nine animals in three independent experiments.

The dose and the way of E2 administration were taken over from the literature as a replacement protocol, in order to achieve the concentration of E2 near physiological ${ }^{(29,30)}$. The dose and timing of insulin treatment were also estimated on the basis of literature data, adjusted to intraperitoneal administration $^{(31)}$, and optimised to observe the peak of changes in the phosphorylation of Akt, Erk and eNOS.

The ovariectomised FFR were compared with the ovariectomised animals subjected to the normal diet to assess the effect of the fructose diet. The FFR treated with E2 were compared with the ovariectomised FFR to analyse the effect of E2 in the context of the fructose diet, as well as with ovariectomised rats on the normal diet, to estimate E2 capacity to counteract to the expected detrimental effect of the FRD. The equivalent comparisons were performed for insulintreated counterparts of all three groups.

\section{Preparation of cardiac cell lysate}

After killing, the heart of rats was removed from the body, washed with cold saline, dried and measured, and the tissue was homogenised on cold with an Ultra-turrax homogeniser in a buffer ( $\mathrm{pH} 7 \cdot 4)$ containing $10 \mathrm{~mm}$-Tris, $150 \mathrm{~mm}$ $\mathrm{NaCl}, 1 \mathrm{~mm}$-EDTA, $1 \mathrm{mm-ethylene} \mathrm{glycol} \mathrm{tetraacetic} \mathrm{acid}$ (EGTA), 1\% Triton X-100, protease inhibitors and phosphatase inhibitors ${ }^{(32)}$. The homogenates were centrifuged at $600 \mathrm{~g}$ for $20 \mathrm{~min}$ at $4^{\circ} \mathrm{C}$ and the supernatants obtained were ultracentrifuged for $60 \mathrm{~min}$ at $100000 \mathrm{~g}$. Protein concentration was determined by the bicinchoninic acid method ${ }^{(33)}$ and after boiling in Laemmli sample buffer, the supernatants were used as cardiac cell lysate for Western blot analysis.

\section{SDS-PAGE and Western blotting}

Cardiac lysate proteins $(50 \mu \mathrm{g} /$ lane $)$ were separated on $7.5 \%$ or $10 \%$ SDS-PAGE and transferred to polyvinylidene fluoride membranes ${ }^{(34)}$. The membranes were blocked with 5\% bovine serum albumin and blotted with an antibody against phospho-Akt, anti-phospho-eNOS or phospho-Erk 1/2. After extensive washing, the membranes were incubated with the appropriate secondary horseradish peroxidase-conjugated antibody and used for subsequent detection with ECL reagents. Thereafter, the membranes were stripped, washed and reprobed with antibodies against Akt, eNOS or Erk, respectively. To ensure that protein loading was equal in all samples, the blots were stripped again and incubated with actin antibody. Films were scanned and analysed using Image J software (NIH).

\section{RNA isolation and quantitative real-time RT-PCR}

Immediately after removal, animal tissue was snap-frozen in liquid $\mathrm{N}_{2}$ and then stored at $-70^{\circ} \mathrm{C}$. Total RNA was 
isolated with TRI Reagent (Ambion, Inc.) according to the manufacturer's instructions. The quantity of mRNA was assessed by a NanoDrop ${ }^{\circledR}$ ND-1000 spectrophotometer (Thermo Scientific). Structural integrity of RNA was confirmed by formaldehyde agarose gel electrophoresis. Total RNA from the tissue specimens with satisfactory quality was converted to complementary DNA. Then, $1 \mu \mathrm{g}$ of RNA was treated with DNase I (Fermentas) and reverse transcription was performed using a First Strand cDNA Synthesis Kit, with oligo-dT18 primers (Fermentas), according to the manufacturer's instructions. Mock reactions lacking RT were performed during the complementary DNA synthesis step in order to additionally exclude genomic contamination. Real-time PCR was performed in duplicate in an ABI Real-time 7500 System (ABI). Detection of eNOS gene expression was done by amplification in a total volume of $25 \mu \mathrm{l}$ in EvaGreen qPCR Mastermix-R (Applied Biological Materials, Inc.). Final concentrations of primers were $6.25 \mu \mathrm{mol} / \mathrm{l}$ each. The reaction conditions were as follows: an initial denaturation at $95^{\circ} \mathrm{C}$ for $10 \mathrm{~min}$ followed by forty cycles of a denaturation step at $95^{\circ} \mathrm{C}$ for $45 \mathrm{~s}$, an annealing step at $60^{\circ} \mathrm{C}$ for $60 \mathrm{~s}$ and an elongation step at $72^{\circ} \mathrm{C}$ for $60 \mathrm{~s}$. All samples were normalised to the housekeeping GAPDH transcript levels. Primer sequences of $G A P D H$ and $e N O S$ genes are available upon request. Differences in mRNA expression between the groups were tested by REST 09 software (Corbett Life Science).

\section{Statistical analysis}

Values are expressed as means and standard deviations (except for mRNA expression results, where standard errors were used instead of standard deviations). We performed the same experiment three times independently and pooled the results for all nine animals per group for one-way ANOVA. A value of $P<0.05$ was considered as statistically significant. The power analysis of the study was performed by 'PS power and sample size program' software ${ }^{(35)}$. The power value for nine animals per group, with an observed effect size and standard deviations and a significance level of 0.05 , was $>0.8$. In addition, differences in mRNA expression between the groups were tested by a pairwise randomisation and bootstrapping technique using the relative expression software tool, REST 09 (Corbett Life Science, http://rest.gene-quantification.info) ${ }^{(36)}$.

\section{Results}

Mass of the heart of fructose-fed rats related to oestradiol status

Based on the data that the FRD and the E2 treatment influence the mass of the heart and are included in the genesis or protection of heart hypertrophy, respectively, we measured the mass of the heart and expressed it as an absolute value or relative to the body mass. As presented in Fig. 1, neither diet nor hormone replacement treatment altered the mass of the heart (Fig. 1(a)) and the heart:body ratio (Fig. 1(b)).
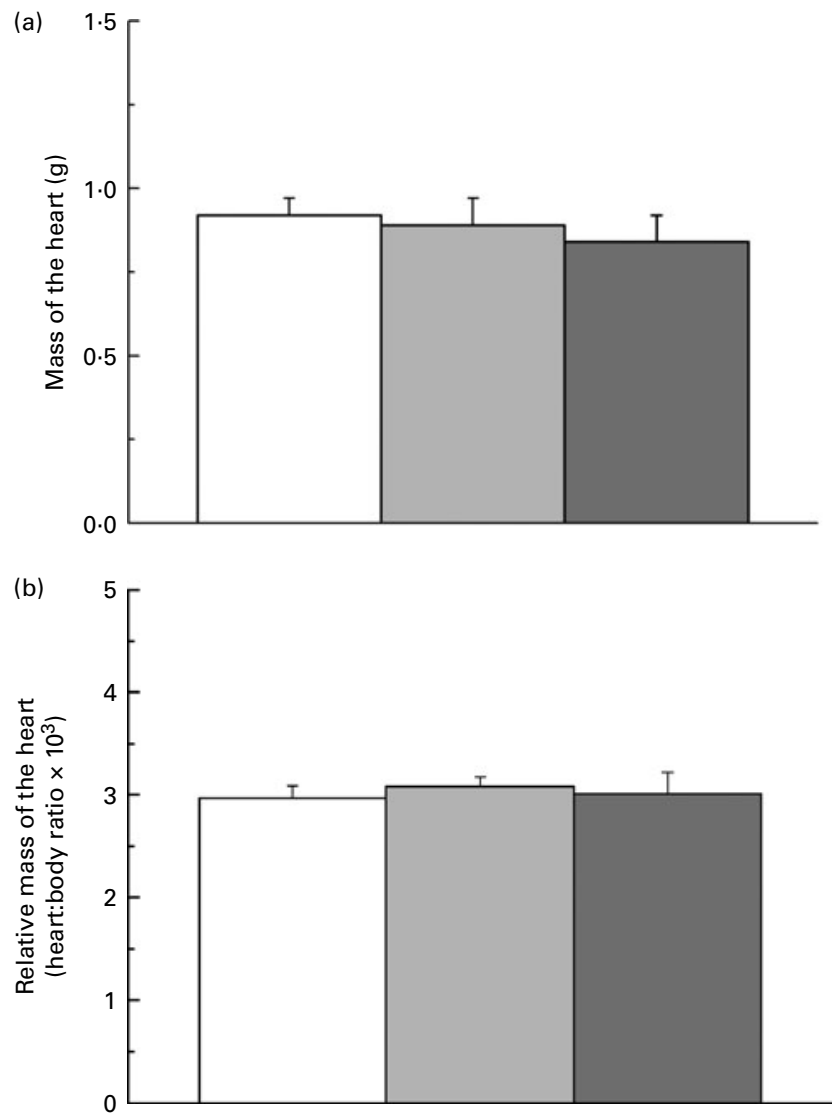

Fig. 1. (a) Absolute and (b) relative mass of the heart of fructose-fed rats: the role of oestradiol. Mass of the heart was measured immediately after killing and isolation from the body. It is expressed as an absolute value and as a heart:body ratio. Values are means of three independent experiments with nine animals per group, with standard deviations represented by vertical bars. OVX, ovariectomy; ND, normal diet; FRD, fructose-rich diet; E2, oestradiol treatment. $\square$, OVX-ND; $\square$, OVX-FRD; $\square$, OVX-FRD + E2.

\section{Effects of the fructose-rich diet and oestradiol status on the expression and phosphorylation of Akt in the heart of ovariectomised female rats}

Akt kinase is regulated by numerous phosphorylations of the molecule, but those at positions $\operatorname{Ser}^{473}$ and $\mathrm{Thr}^{308}$ are assigned as crucial for enzyme activity. As presented in Fig. 2(a) and (b), the results of the present study confirmed that insulin increases the phosphorylation of both residues. The FRD decreased the phosphorylation of both amino acid residues in the heart of rats, in basal conditions (with no additional insulin treatment) and/or insulin-stimulated conditions $\left(P<0.001\right.$, OVX-FRD $v$. OVX-ND for $\operatorname{Ser}^{473} ; P<0 \cdot 01$, OVXFRD $v$. OVX-ND; $P<0.05$, OVX-FRD + insulin $v$. OVX-ND + insulin for $\mathrm{Thr}^{308}$; Fig. 2(a) and (b)). The E2 treatment of FFR reversed phospho-Ser ${ }^{473}$ Akt content to the control level in basal conditions. However, E2 replacement was effective in reversing the diet effects on Akt phosphorylation at $\mathrm{Thr}^{308}$ in insulin-stimulated conditions, while failed to reverse the FRD effect in basal condition $(P<0 \cdot 01$, OVX-FRD + E2 $v$. OVX-ND). Neither the diet nor hormone replacement changed the protein expression of Akt in the heart (Fig. 2(c)), determined as Akt content in cardiac lysate. 


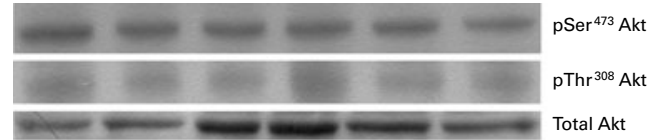

OVX-ND OVX-ND OVX-FRD OVX-FRD OVX-FRD OVX-FRD +INS + INS + E2 + E2+INS
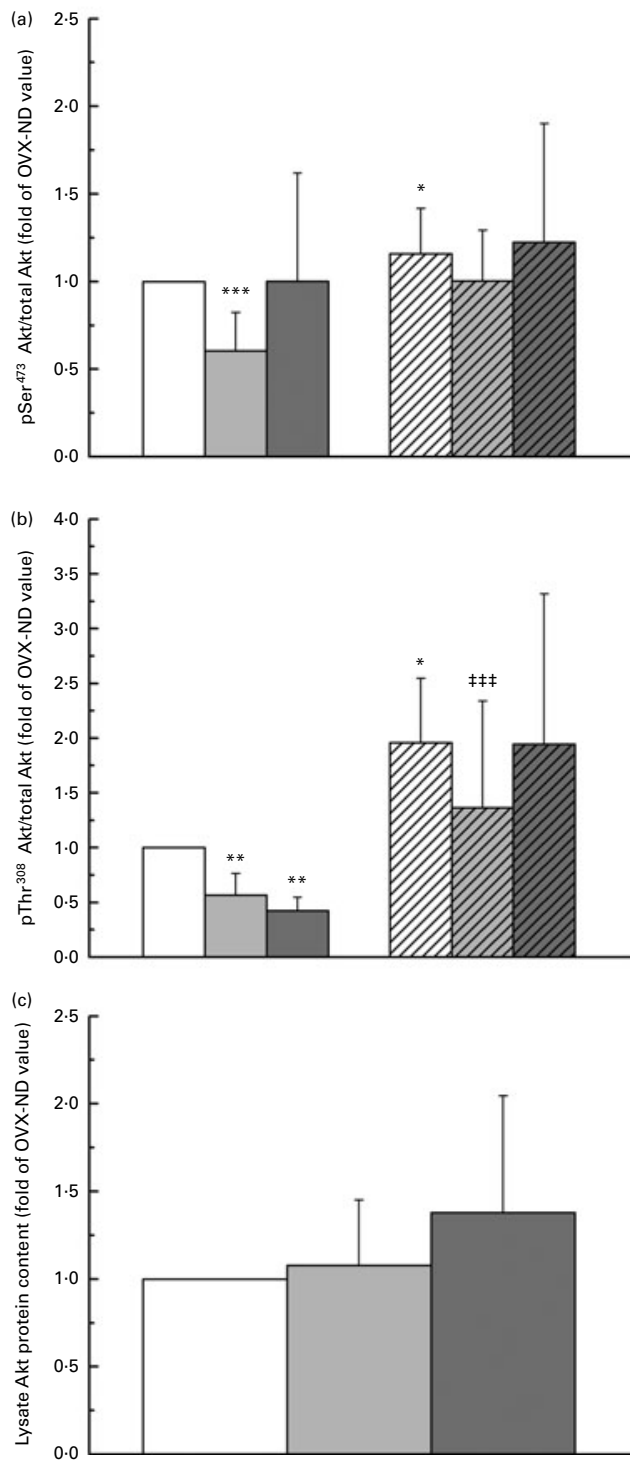

Fig. 2. Alterations of cardiac Akt phosphorylation at (a) $\operatorname{Ser}^{473}\left(\mathrm{pSer}^{473}\right)$, (b) $\mathrm{Thr}^{308}\left(\mathrm{pThr}{ }^{308}\right)$ and (c) protein expression in fructose-fed rats in the absence or presence of oestradiol. Protein samples of the cardiac cell lysate of ovariectomised female rats on the normal or fructose-enriched diet, with or without oestradiol (E2) replacement, were analysed by the Western blot method using an antibody against Akt or Akt phosphorylated at $\mathrm{Ser}^{473} \mathrm{or} \mathrm{Thr}^{308}$. Three independent experiments with a total of nine rats per group were quantified. Phospho-Akt content was normalised to total Akt. Results are expressed as a fold of appropriate control value (ovariectomised rats on normal diet). Values are means, with standard deviations represented by vertical bars. Representative Western blots are also shown. OVX, ovariectomy; ND, normal diet; FRD, fructose-rich diet; INS, insulin treatment. Mean value was significantly different from that of the OVX-ND group: ${ }^{*} P<0.05$. Mean value was significantly different from that of the OVX-ND group: ${ }^{\star *} P<0.01$. Mean value was significantly different from that of the OVX-ND group: ${ }^{* \star *} P<0.001$. Mean value was significantly different from that of the OVX-ND + INS group: $\ddagger P<0.05$. $\square$, OVX-ND;

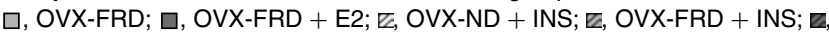
OVX-FRD + E2 + INS
Expression and phosphorylation of the cardiac endothelial nitric oxide synthase effects of the fructose-rich diet and oestradiol replacement

Enzyme activity of eNOS is also regulated by a number of serine/threonine phosphorylations. Phosphorylation at serine 1177 is accompanied by the activation of eNOS, while phosphorylation at threonine 495 is found to be constitutive and inhibitory. Insulin itself stimulated phosphorylation at Ser ${ }^{1177}$ only (Fig. 3(a)). As presented in Fig. 3(a), a significant decrease in $\operatorname{Ser}^{1177}$ phosphorylation of eNOS was observed in the heart of FFR, in insulin-stimulated conditions $(P<0.05$, OVX-FRD + insulin $v$. OVX-ND + insulin). E2 over-reverted the negative effects of the fructose diet $(P<0.05$, OVXFRD + E2 $v$. OVX-FRD; $P<0 \cdot 05$, OVX-FRD + E2 $v$. OVX-ND; $P<0 \cdot 01, \quad$ OVX-FRD + E2 + insulin $v$. OVX-FRD + insulin; $P<0 \cdot 05$, OVX-FRD $+\mathrm{E} 2+$ insulin $v$. OVX-ND + insulin) and elevated the eNOS serine 1177 phosphorylation level above the level detected in rats fed on the normal diet.

In contrast to the serine 1177 site, the FRD caused an increase in the phosphorylation of eNOS at threonine 495 in basal $(P<0 \cdot 001$, OVX-FRD $v$. OVX-ND) and insulin-stimulated conditions $(P<0 \cdot 05$, OVX-ND + insulin $v$. OVX-ND + insulin $)$ (Fig. 3(b)). E2 replacement had no effect on this phosphorylation in FFR.

The protein content of eNOS determined in heart lysate was unaffected by the FRD and did not depend on the presence of E2 (Fig. 3(c)). In contrast to protein expression, as presented in Fig. 3(d), the steady-state mRNA level of eNOS was surprisingly elevated in FFR (OVX-FRD $v$. OVX-ND, expression mean factor $5 \cdot 765$, sE range $2 \cdot 355-12 \cdot 795$, 95\% CI $1.904,19 \cdot 051, P=0.000)$. It is interesting to note that OVX and the fructose diet separately acted as strong negative regulators of cardiac eNOS mRNA expression (ovariectomised $v$. intact rats, on the normal diet: mean factor $0 \cdot 154, P=0 \cdot 018$; intact rats on the fructose diet $v$. intact rats on the normal diet: mean factor $0.077, P=0 \cdot 016)$. In FFR, E2 replacement returned the eNOS steady-state mRNA level to the control value (OVX-FRD + E2 $v$. OVX-ND, expression mean factor $0 \cdot 995$, se range $0 \cdot 290-3 \cdot 144,95 \%$ CI $0 \cdot 193,4 \cdot 620, P=0 \cdot 901)$.

Effects of the fructose-rich diet and oestradiol replacement on the phosphorylation and expression of extracellular signal-regulated kinase $1 / 2$ in the heart of ovariectomised female rats

The absence of the potential effects of the fructose diet and E2 treatment on heart mass initiated the analysis of their effects on mitogenic signalling through Erk $1 / 2$ kinases. In accordance with the results obtained for the absolute and relative mass of the heart, we did not observe any changes in Erk 1/ 2 phosphorylation at $\mathrm{Thr}^{202} / \mathrm{Tyr}^{204}$ and protein expression, caused by the diet or hormone treatment (Fig. 4(a) and (b)).

\section{Discussion}

Akt and eNOS play a very important role in the regulation of cardiac functions ${ }^{(10,12)}$. Cardiac Akt/eNOS signalling is a 
target of insulin resistance ${ }^{(6)}$ and E2 regulation as well ${ }^{(16)}$. Phosphorylation of eNOS by Akt with a subsequent increase in NO production is an important downstream effector in the anti-apoptotic signalling by insulin. Moreover, increasing evidence suggests that eNOS acts as a metabolic sensor in cardiomyocytes, implying that defective NO production might be linked to cardiomyocyte metabolism dysfunction ${ }^{(18)}$. On the basis of published data, we performed the study of the effects of oestrogen replacement on this signalling pathway in ovariectomised female rats fed the FRD. In addition, we analysed heart mass and the mitogenic Erk pathway, which also might be modulated by the diet regimen and oestrogen treatment.

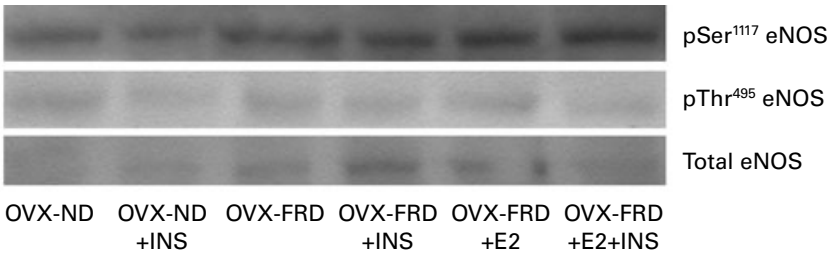

(a)

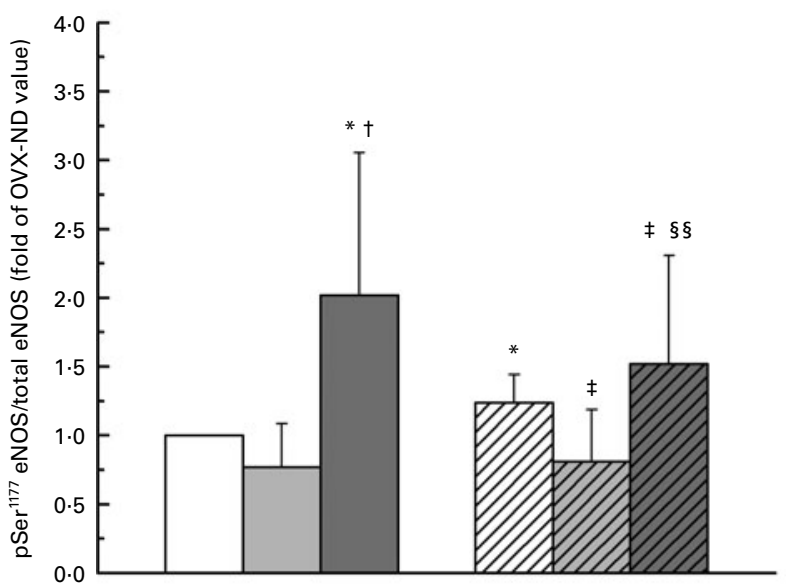

(c)

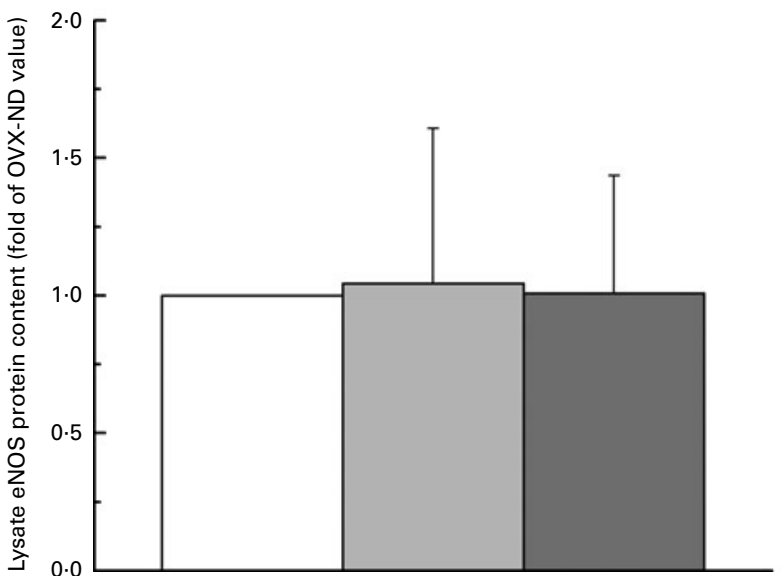

(b)

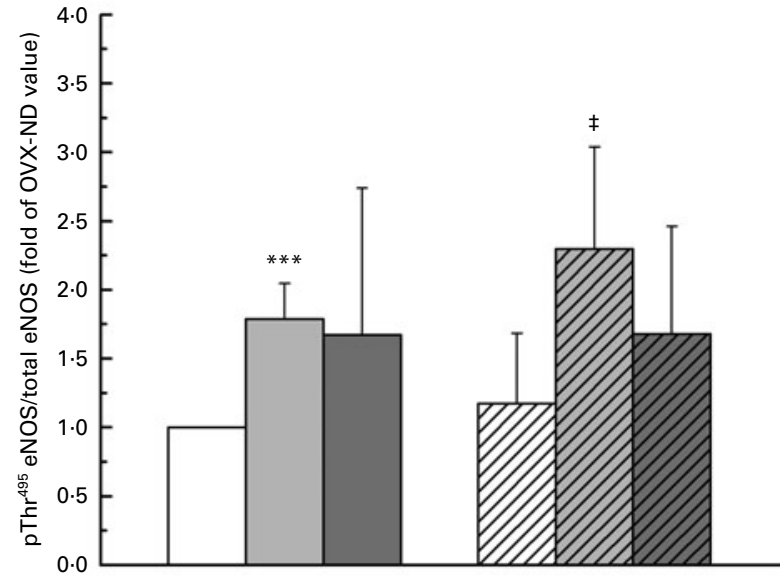

(d)

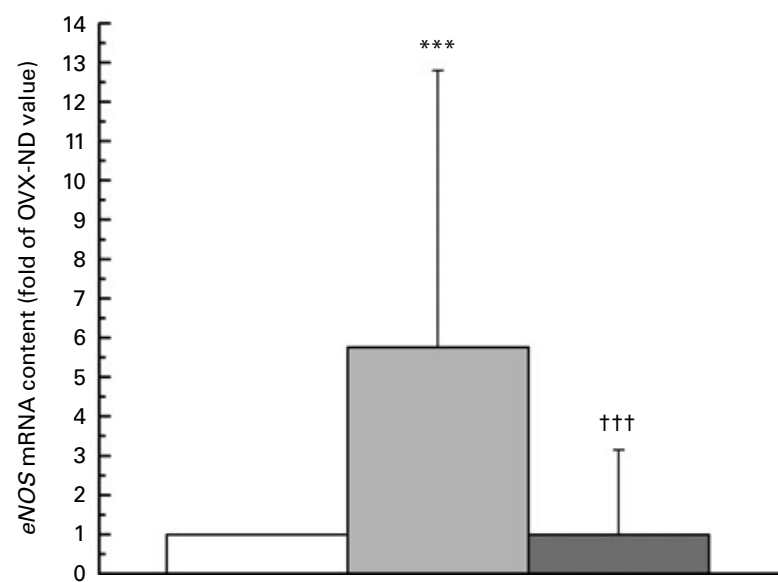

Fig. 3. Changes in the phosphorylation of endothelial nitric oxide synthase (eNOS) at (a) Ser ${ }^{1177}\left(\mathrm{pSer}^{1177}\right)$, (b) Thr ${ }^{495}$ (pThr ${ }^{495}$ ) and (c) eNOS protein expression and (d) steady-state eNOS mRNA level in the heart of rats on the fructose-enriched diet: the role of oestradiol. Protein samples of cardiac cell lysate were resolved on $7.5 \%$ SDS-PAGE and analysed by the Western blot method using an antibody raised against eNOS or eNOS phosphorylated at Ser ${ }^{1177}$ or Thr ${ }^{495}$. Three independent experiments with a total of nine rats per group were quantified. Phospho-eNOS content was normalised to total eNOS. Results are expressed as a fold of appropriate control value (ovariectomised rats on the normal diet). Values are means, with standard deviations represented by vertical bars. Representative Western blots are also shown. OVX, ovariectomy; ND, normal diet; INS, insulin treatment; FRD, fructose-rich diet; E2, oestradiol treatment. Mean value was significantly different from that of the OVX-ND group: ${ }^{*} P<0.05$. Mean value was significantly different from that of the OVX-ND group: ${ }^{* * *} P<0.001$. Mean value was significantly different from that of the OVX-FRD group: $\dagger P<0.05$. Mean value was significantly different from that of the OVX-FRD group: $\dagger+\dagger P<0.001$. Mean value was significantly different from that of the OVX-ND + INS group: $\ddagger P<0.05$. Mean value was significantly different from that of the OVX-FRD + INS

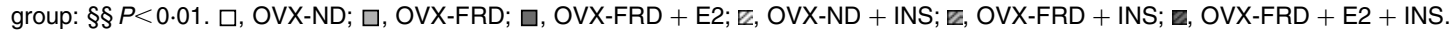




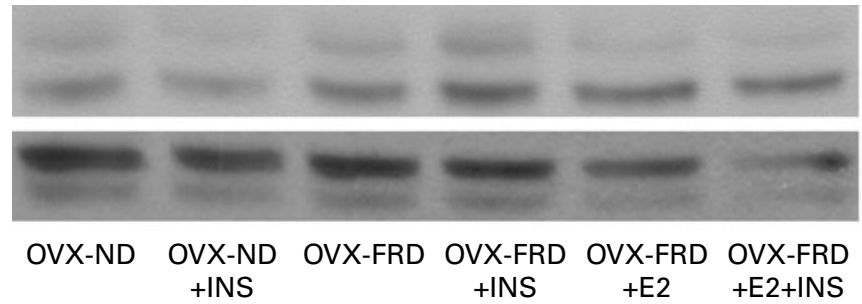

(a)
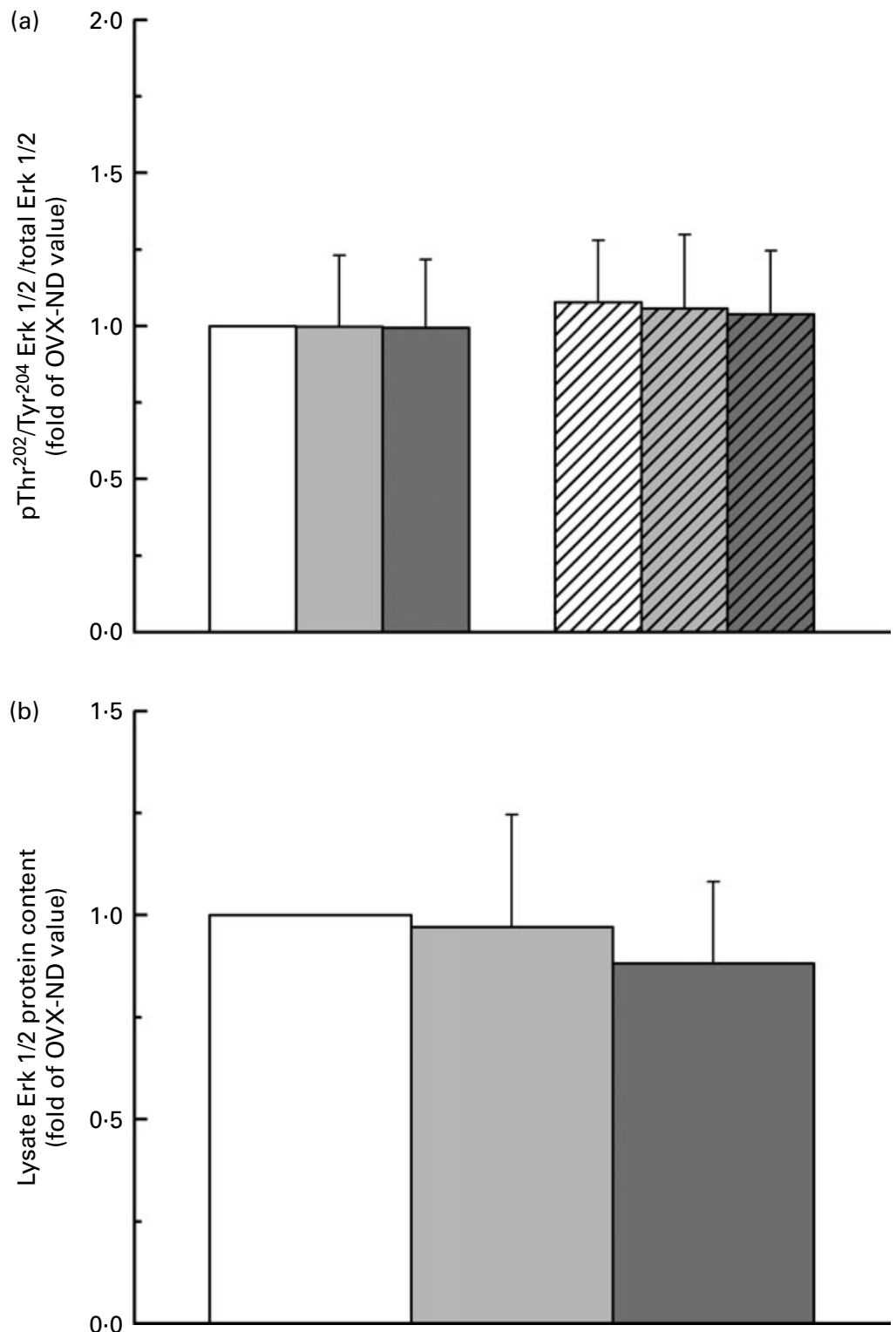

Fig. 4. Effects of the fructose-rich diet and oestradiol treatment on cardiac (a) Erk $1 / 2 \mathrm{Thr}^{202} / \mathrm{Tyr}^{204}$ phosphorylation level and (b) Erk $1 / 2$ protein expression The content of total Erk $1 / 2$ and $\left.\mathrm{pThr}^{202}\right) / \mathrm{Tyr}^{204} \mathrm{Erk} 1 / 2$ in the lysate of cardiac cells was analysed by Western blot using a specific antibody raised against Erk $1 / 2$ or Erk $1 / 2$ phosphorylated at $\mathrm{Thr}^{202} / \mathrm{Tyr}^{204}$. Three independent experiments with a total of nine rats per group were quantified. Phospho-Erk $1 / 2$ content was normalised to total Erk 1/2. Results are expressed as a fold of appropriate control value (ovariectomised rats on the normal diet). Values are means, with standard deviations represented by vertical bars. Representative Western blots are also shown. OVX, ovariectomy; ND, normal diet; INS, insulin treatment; FRD, fructoserich diet; E2, oestradiol treatment. $\square$, OVX-ND; $\square$, OVX-FRD; $\square$, OVX-FRD + E2; $\mathbb{z}$, OVX-ND + INS; $\mathbb{z}$, OVX-FRD + INS; z, OVX-FRD + E2 + INS. 
In contrast to studies implicating fructose effects on the development of heart hypertrophy ${ }^{(21-23)}$, we did not observe any changes in absolute and relative heart mass in FFR, which could be attributed to the duration of the diet regimen and/or the content of fructose in the applied diet ${ }^{(21,23)}$. Hormone replacement treatment was also ineffective in terms of the changes in the heart mass of FFR. These data are in line with the results obtained for the mitogenic Erk 1/2 signalling pathway regulating cell proliferation and growth, where diet- or hormone-related changes were absent. The lack of the fructose effect on Erk 1/2 phosphorylation is in accordance with our recently reported results ${ }^{(6)}$, but the absence of the E2 effect is in contrast to the results obtained in cell culture $^{(24)}$ or in acute hormone treatment ${ }^{(25)}$. It could be a consequence of the way of hormone administration, but also might implicate some fructose diet-induced disturbances in the activation of Erk 1/2 signalling by E2.

The results of the present study concerning the deregulation of cardiac Akt/eNOS signalling in FFR are mostly in accordance with literature data. As we already reported, the fructose-enriched diet decreased cardiac Akt phosphorylation at Ser ${ }^{473}$ and $\mathrm{Thr}^{308}$ and eNOS at Ser ${ }^{1177}$, especially in insulinstimulated conditions ${ }^{(6)}$. On the other hand, increased intake of fructose stimulated inhibitory phosphorylation of cardiac eNOS at threonine 495. It was well documented that heart eNOS activity is decreased in $\mathrm{FFR}^{(5,22,37,38)}$. Myocardial Akt protein expression and its $\operatorname{Ser}^{473}$ phosphorylation, as well as eNOS expression and phosphorylation at $\mathrm{Ser}^{1177}$, were decreased in $\mathrm{FFR}^{(39)}$. However, in contrast to the present results, expression of eNOS protein and mRNA was shown to be reduced in the heart of $\mathrm{FFR}^{(40)}$. It was astounding that we observed no effect of the fructose diet on eNOS protein content, but a strong increase in the gene expression. We have no physiological explanation particularly regarding that OVX and fructose, in the present study, separately caused a strong decrease in cardiac eNOS mRNA level.

There are no published data concerning the effects of E2 on cardiac Akt/eNOS signalling in increased fructose intake conditions or other animal models of insulin resistance. The only relevant results that can be cited are those describing the effects of $\mathrm{E} 2$ treatment in normal rats. We recently reported a rapid stimulatory effect of a single injection of E2 on cardiac Akt Ser ${ }^{473}$ and $\mathrm{Thr}^{308}$, as well as on eNOS Ser ${ }^{1177}$ phosphorylation $^{(41)}$. Others have also observed that E2 treatment increased Akt phosphorylation and Akt-mediated eNOS phosphorylation $\left(\operatorname{Ser}^{1177}\right)^{(42)}$. A number of studies have shown that E2 increased cardiac levels of eNOS, and phosphorylated eNOS $\left(S^{1177}\right)$, thus exerting potentially beneficial cardiovascular effects of $\mathrm{NO}^{(43,44)}$. In ovariectomised rats, E2 replacement restored eNOS activity and expression ${ }^{(45)}$. E2 stimulated the expression of eNOS in both neonatal and adult cardiac myocytes ${ }^{(15)}$. In the present study, we observed no effect of E2 on the expression of eNOS in the heart of FFR and its threonine 495 phosphorylation, while the hormone effect on serine 1177 phosphorylation was strongly inducible.

We noted that the effect of the FRD and the ability of E2 to exert an effect on phosphorylation of different amino acid residues in Akt and eNOS are selective. Observed differences in the effects of fructose and E2 on the phosphorylation level of different amino acids in the protein structure of Akt and eNOS actually implicate the different effects of these agents on kinase/phosphatase pathways responsible for the phosphorylation/dephosphorylation of these sites. It is well known that phosphorylation of Akt $\mathrm{Thr}^{308}$ is attributed to phosphoinositide-dependent protein kinase 1 (PKD1) kinase, while Rictor-mammalian target of repamycin (mTOR) kinase is probably responsible for $\operatorname{Ser}^{473}$ phosphorylation ${ }^{(19)}$. On the other hand, eNOS is a substrate of Akt kinase that phosphorylates the Ser ${ }^{1177}$ position ${ }^{(46)}$. The phosphatase to which is referred insulin-induced dephosphorylation of eNOS at the negative regulatory site $\mathrm{Thr}^{495}$ is probably protein phosphatase $1^{(20)}$.

To the best of our knowledge, no published literature contains any data concerning the effects of enhanced fructose intake on kinases/phosphatases involved in the phosphorylation/dephosphorylation of Akt and eNOS molecules on amino acid residues analysed in the present study, except Akt as a kinase responsible for eNOS phosphorylation at Ser ${ }^{1177}$. The FRD-induced decrease in Akt phosphorylation points to the decreased activity of responsible kinases or the increased activity of phosphatases that counteract PDK1 and Rictor-mTOR. Inhibition of Akt could be a direct explanation of the observed decrease in the phosphorylation of eNOS at $\mathrm{Ser}^{1177}$ in FFR. The increase in the phosphorylation of eNOS at $\mathrm{Thr}^{495}$ in animals fed on the FRD indicates changes in the activity of protein phosphatase 1 or kinases responsible for the phosphorylation of this amino acid residue.

Data concerning the effects of E2 on these kinases and phosphatases, particularly in cardiac tissue, are also extremely scarce and we can only speculate, on the basis of the present results, that E2 activates cardiac Rictor-mTOR kinase and probably PDK1, but has no effect on protein phosphatase 1 in the heart.

In conclusion, there was a consistent suppression of cardiac Akt/eNOS signalling at the level of protein phosphorylation in FFR. It is interesting to note that E2 managed to abrogate some, but not all, of fructose-induced damage at the basal level or in insulin-stimulated conditions, implicating its selective effects on kinase and phosphatase pathways involved in the activation of Akt/eNOS signalling. As a matter of future perspectives, it would be very intriguing to conduct an additional study the effects of E2 on PDK1, Rictor-mTOR and protein phosphatase 1 in cardiac tissue.

\section{Acknowledgements}

This study was supported by project grant no. 41009 and project grant no. 175085, from the Ministry of Education and Science, Republic of Serbia. S. R. conducted the experiments and analysed the data. G. K. and Z. Z. designed the study, conducted the experiments, analysed the data and wrote the paper. S. T., T. M., M. S. and M. P. conducted the experiments and statistical analysis. M. Z. and A. S. conducted the experiments and revised the manuscript. All authors contributed to and approved the final manuscript. The authors declare that there are no conflicts of interest. 


\section{References}

1. de Moura RF, Ribeiro C, de Oliveira JA, et al. (2009) Metabolic syndrome signs in Wistar rats submitted to different high-fructose ingestion protocols. Br J Nutr 101, 1178-1184.

2. Tran LT, Yuen VG \& McNeill JH (2009) The fructose-fed rat: a review on the mechanisms of fructose-induced insulin resistance and hypertension. Mol Cell Biochem 332, 145-159.

3. Axelsen LN, Lademann JB, Petersen JS, et al. (2010) Cardiac and metabolic changes in long-term high fructose-fat fed rats with severe obesity and extensive intramyocardial lipid accumulation. Am J Physiol Regul Integr Comp Physiol 298, R1560-R1570.

4. Dekker MJ, Su Q, Baker C, et al. (2010) Fructose: a highly lipogenic nutrient implicated in insulin resistance, hepatic steatosis, and the metabolic syndrome. Am J Physiol Endocrinol Metab 299, E685-E694.

5. Miatello R, Risler N, Castro C, et al. (2001) Aortic smooth muscle cell proliferation and endothelial nitric oxide synthase activity in fructose-fed rats. Am J Hypertens 14, $1135-1141$.

6. Zakula Z, Koricanac G, Tepavcevic S, et al. (2011) Impairment of cardiac insulin signaling in fructose-fed ovariectomized female Wistar rats. Eur J Nutr 50, 543-551.

7. Galipeau D, Verma S \& McNeill JH (2002) Female rats are protected against fructose-induced changes in metabolism and blood pressure. Am J Physiol Heart Circ Physiol 283, $\mathrm{H} 2478-\mathrm{H} 2484$.

8. Brown L, Hoong I \& Doggrell SA (2000) The heart as a target for oestrogens. Heart Lung Circ 9, 113-125.

9. Patten RD \& Karas RH (2006) Estrogen replacement and cardiomyocyte protection. Trends Cardiovasc Med 16, 69-75.

10. Massion PB \& Balligand JL (2003) Modulation of cardiac contraction, relaxation and rate by the endothelial nitric oxide synthase (eNOS): lessons from genetically modified mice. J Physiol 546, 63-75.

11. Massion PB, Feron O, Dessy C, et al. (2003) Nitric oxide and cardiac function: ten years after, and continuing. Circ Res $\mathbf{9 3}$, 388-398.

12. Matsui T, Nagoshi T \& Rosenzweig A (2003) Akt and PI3kinase signaling in cardiomyocyte hypertrophy and survival. Cell Cycle 2, 220-223.

13. Camper-Kirby D, Welch S, Walker A, et al. (2001) Myocardial Akt activation and gender: increased nuclear activity in females versus males. Circ Res 88, 1020-1027.

14. Murphy E \& Steenbergen C (2007) Cardioprotection in females: a role for nitric oxide and altered gene expression. Heart Fail Rev 12, 293-300.

15. Nuedling S, Kahlert S, Loebbert K, et al. (1999) 17 Betaestradiol stimulates expression of endothelial and inducible NO synthase in rat myocardium in-vitro and in-vivo. Cardiovasc Res 43, 666-674.

16. Patten RD, Pourati I, Aronovitz MJ, et al. (2004) 17Betaestradiol reduces cardiomyocyte apoptosis in vivo and in vitro via activation of phospho-inositide-3 kinase/Akt signaling. Circ Res 95, 692-699.

17. Haynes MP, Sinha D, Russell KS, et al. (2000) Membrane estrogen receptor engagement activates endothelial nitric oxide synthase via the PI3-kinase-Akt pathway in human endothelial cells. Circ Res 87, 677-682.

18. Yu Q, Gao F \& Ma XL (2011) Insulin says NO to cardiovascular disease. Cardiovasc Res 89, 516-524.

19. Sale EM \& Sale GJ (2008) Protein kinase B: signalling roles and therapeutic targeting. Cell Mol Life Sci 65, 113-127.

20. Andreozzi F, Laratta E, Procopio C, et al. (2007) Interleukin-6 impairs the insulin signaling pathway, promoting production of nitric oxide in human umbilical vein endothelial cells. Mol Cell Biol 27, 2372-2383.

21. Al-Awwadi NA, Araiz C, Bornet A, et al. (2005) Extracts enriched in different polyphenolic families normalize increased cardiac NADPH oxidase expression while having differential effects on insulin resistance, hypertension, and cardiac hypertrophy in high-fructose-fed rats. J Agric Food Chem 53, 151-157.

22. Miatello R, Vazquez M, Renna N, et al. (2005) Chronic administration of resveratrol prevents biochemical cardiovascular changes in fructose-fed rats. Am J Hypertens $\mathbf{1 8}$ 864-870.

23. Panchal SK, Poudyal H, Iyer A, et al. (2011) High-carbohydrate, high-fat diet-induced metabolic syndrome and cardiovascular remodeling in rats. J Cardiovasc Pharmacol 57, 611-624.

24. de Jager T, Pelzer T, Muller-Botz S, et al. (2001) Mechanisms of estrogen receptor action in the myocardium. Rapid gene activation via the ERK1/2 pathway and serum response elements. J Biol Chem 276, 27873-27880.

25. Koricanac G, Tepavcevic S, Zakula Z, et al. (2011) Interference between insulin and estradiol signaling pathways in the regulation of cardiac eNOS and $\mathrm{Na}(+) / \mathrm{K}(+)$-ATPase. Eur J Pharmacol 655, 23-30.

26. Pedram A, Razandi M, Lubahn D, et al. (2008) Estrogen inhibits cardiac hypertrophy: role of estrogen receptor-beta to inhibit calcineurin. Endocrinology 149, 3361-3369.

27. Donaldson C, Eder S, Baker C, et al. (2009) Estrogen attenuates left ventricular and cardiomyocyte hypertrophy by an estrogen receptor-dependent pathway that increases calcineurin degradation. Circ Res 104, 265 275, 211p following 275 .

28. Vasudevan H, Xiang H \& McNeill JH (2005) Differential regulation of insulin resistance and hypertension by sex hormones in fructose-fed male rats. Am J Physiol Heart Circ Physiol 289, H1335-H1342.

29. Busserolles J, Mazur A, Gueux E, et al. (2002) Metabolic syndrome in the rat: females are protected against the prooxidant effect of a high sucrose diet. Exp Biol Med (Maywood) 227, 837-842.

30. Sales S, Ureshino RP, Pereira RT, et al. (2010) Effects of 17beta-estradiol replacement on the apoptotic effects caused by ovariectomy in the rat hippocampus. Life Sci $\mathbf{8 6}$, 832-838.

31. Carvalheira JB, Calegari VC, Zecchin HG, et al. (2003) The cross-talk between angiotensin and insulin differentially affects phosphatidylinositol 3-kinase- and mitogen-activated protein kinase-mediated signaling in rat heart: implications for insulin resistance. Endocrinology 144, 5604-5614.

32. Dupont J, Derouet M, Simon J, et al. (1998) Nutritional state regulates insulin receptor and IRS-1 phosphorylation and expression in chicken. Am J Physiol 274, E309-E316.

33. Smith PK, Krohn RI, Hermanson GT, et al. (1985) Measurement of protein using bicinchoninic acid. Anal Biochem 150, 76-85.

34. Laemmli UK (1970) Cleavage of structural proteins during the assembly of the head of bacteriophage T4. Nature $\mathbf{2 2 7}$, 680-685.

35. Dupont WD \& Plummer WD Jr (1990) Power and sample size calculations. A review and computer program. Control Clin Trials 11, 116-128.

36. Pfaffl MW, Horgan GW \& Dempfle L (2002) Relative expression software tool (REST) for group-wise comparison and statistical analysis of relative expression results in realtime PCR. Nucleic Acids Res 30, e36. 
37. Vazquez-Prieto MA, Rodriguez Lanzi C, Lembo C, et al. (2011) Garlic and onion attenuates vascular inflammation and oxidative stress in fructose-fed rats. J Nutr Metab 2011, 475216

38. Vazquez-Prieto MA, Renna NF, Lembo C, et al. (2010) Dealcoholized red wine reverse vascular remodeling in an experimental model of metabolic syndrome: role of NAD(P)H oxidase and eNOS activity. Food Funct 1, 124-129.

39. Prakash P, Khanna V, Singh V, et al. (2011) Atorvastatin protects against ischemia-reperfusion injury in fructoseinduced insulin resistant rats. Cardiovasc Drugs Ther 25, 285-297.

40. Nyby MD, Matsumoto K, Yamamoto K, et al. (2005) Dietary fish oil prevents vascular dysfunction and oxidative stress in hyperinsulinemic rats. Am J Hypertens 18, 213-219.

41. Tepavcevic S, Koricanac G, Zakula Z, et al. (2011) Interaction between insulin and estradiol in regulation of cardiac glucose and free fatty acid transporters. Horm Metab Res $\mathbf{4 3}$, $524-530$.
42. Tagashira H, Bhuiyan S, Shioda N, et al. (2011) Distinct cardioprotective effects of 17beta-estradiol and dehydroepiandrosterone on pressure overload-induced hypertrophy in ovariectomized female rats. Menopause 18, 1317-1326.

43. Kan WH, Hsu JT, Ba ZF, et al. (2008) p38 MAPK-dependent eNOS upregulation is critical for 17 beta-estradiol-mediated cardioprotection following trauma-hemorrhage. Am J Physiol Heart Circ Physiol 294, H2627-H2636.

44. Ricchiuti V, Lian CG, Oestreicher EM, et al. (2009) Estradiol increases angiotensin II type 1 receptor in hearts of ovariectomized rats. $J$ Endocrinol 200, 75-84.

45. Wang X \& Abdel-Rahman AA (2002) Estrogen modulation of eNOS activity and its association with caveolin-3 and calmodulin in rat hearts. Am J Physiol Heart Circ Physiol 282, $\mathrm{H} 2309-\mathrm{H} 2315$.

46. Michell BJ, Griffiths JE, Mitchelhill KI, et al. (1999) The Akt kinase signals directly to endothelial nitric oxide synthase. Curr Biol 9, 845-848. 